\title{
Barley Seedlings in Response to Salinity and Artemisinin Stress in the Present of Freeze-Thaw Stress
}

Zhang Wei ( $\sim$ baogz@jlu.edu.cn )

Jilin University

Guozhang Bao

Jilin University https://orcid.org/0000-0003-0329-8001

Tang Wenyi

Jilin University

Dai Gejun

Jilin University

Xiao Jing

Jilin University

Liu Jiapeng

Jilin University

Wang Zhao

Jilin University

Xi Jinghui

Jilin University

\section{Research Article}

Keywords: Allelochemicals, Photosynthesis, Qinghai-Tibet Plateau, Soil salinization, Physiological effects, freeze-thaw

Posted Date: January 3rd, 2022

DOI: https://doi.org/10.21203/rs.3.rs-1034052/v2

License: (c) (i) This work is licensed under a Creative Commons Attribution 4.0 International License.

Read Full License 


\section{Abstract}

In the Qinghai-Tibet Plateau, both the large daily temperature difference and soil salinization make plants susceptible to abiotic stresses such as freeze-thaw and salinity. Meanwhile, crops in this area could be subjected to the influence of artemisinin, an allelochemical exuded by Artemisia annua. In the context of freeze-thaw and salinity stresses, artemisinin was induced as an allelopathy stress factor to explore the physiological response of highland barley, including the relative electrical conductivity (RC), soluble protein (SP) content, malondialdehyde (MDA) content, antioxidant enzyme activity, and water use efficiency (WUE).There data suggested that artemisinin weakened the self-osmotic adjustment ability of seedlings, reducing the SOD activity in scavenging efficiency of reactive oxygen species, then causing oxidative damage to cell membrane of seedlings, which significantly increases the content of RC and MDA. Artemisinin stress can reduce the WUE of seedlings and weaken the photosynthesis intensity of seedlings as well. In a word, salinity stress and artemisinin respectively showed a synergistic compound relationship with freeze-thaw stress,

\section{Introduction}

Known as the "Roof of the World", the Qinghai-Tibet Plateau is very sensitive to climate change. In addition, complex geographical and climatic conditions make seasonal frozen soil being widely distributed on the plateau (Wang et al, 2019), resulted from which, the freeze-thaw stress not only affects the function of cell membranes and osmotic adjustment of plant (Bertrand et al, 2016; Min et al, 2020),but also affect the photosynthesis of the above-ground parts of plants through changing soil nutrients, resulting in a decline in vegetation productivity (Domisch et al, 2019; Arfan et al, 2019). The evaporation in the Qinghai-Tibet Plateau generally exceeds the actual precipitation, accelerating the accumulation of salt to the soil surface (Guo 2021),besides, improper agricultural production measures like an increasing amount of chemical fertilizers usage have resulted in the secondary salinization of soil more and more serious (Che et al, 2021). It has been reported that massive salt in the soil could easily cause osmotic stress, ion toxicity and metabolic hindrance to plants, which therefore affects mostly important life processes of plants (Zhang et al, 2021). Recently, the artemisinin secreted by Artemisia annua that is one of the wild medicinal resources in the Qinghai-Tibet area (Ye et al, 2014) would cause allelopathy to the plants growing around it (Herrmann et al, 2013; Hussain and Reigosa, 2014). Studies have shown that a higher concentration of artemisinin solution can inhibit the germination of various crop seeds, reducing photosynthetic rate, root vitality and protective enzyme activities at the seedling stage of crops (Yan et al, 2018).

Highland barley (Hordeum vulgare Linn. var. nudum Hook.f.) is a cereal crop of the gramineous barley genus, and is the main crop in Tibet and Qinghai (Qian et al, 2019). In this paper, highland barley seeds of variety Beiqing No.3 were obtained commercially in the market from an agricultural seed company Sichuan Tianlu Agricultural Science and Technology Development CO.,LTD and used as the experimental material subjected to the combined stresses of salinity salt and artemisinin. Artificially simulated freezethaw experiment was carried out to investigate the physiological responses of barley to freeze-thaw 
stress, salinity stress and allelopathic stress through measuring relative electrical conductivity, soluble protein content, malondialdehyde content, antioxidant enzyme activity and water use efficiency.

\section{Material And Method}

\subsection{Plant culture and compound treatments of salinity and artemisinin}

The full-grained highland barley seeds were selected and disinfected with $0.1 \% \mathrm{KMnO}_{4}$ solution for $2 \mathrm{~h}$, after which the seeds were rinsed with deionized water until the water becoming clear. Then we spread 120 seeds evenly on each of 8 culture dishes randomly named SAF, SOF, OAF, OOF, SAO, SOO, OAO and 000 (Table 1). The artemisinin powder (purity $\geq 98 \%$ ) was purchased from Shaanxi Sciphar Natural Products Co., Ltd. Dissolve $20 \mathrm{mg}$ artemisinin powder in $1 \mathrm{ml}$ acetone to prepare stock solution, then stock solution was diluted to get a concentration of $20 \mathrm{mg} / \mathrm{L}$ artemisinin solution (A solution) with $1 / 2$ Hoagland nutrient solution (Yan et al, 2015). $\mathrm{NaHCO}_{3}$ was added into $1 / 2$ Hoagland nutrient solution and $20 \mathrm{mg} / \mathrm{L}$ artemisinin solution respectively, to get a $60 \mathrm{mM}$ salinity stress solution (S solution) and $60 \mathrm{mM}$ compound stress solution of salinity and artemisinin (S-A solution) prepared.

$500 \mathrm{ml}$ S-A solution was added to the cultivated dishes of Groups SAF and SAO at the same time, and $500 \mathrm{ml} S$ solution for Groups SOF and SOO, $500 \mathrm{ml}$ A solution for Groups OAF and OAO, 500 $\mathrm{ml} 1 / 2$ Hoagland nutrient solution for Groups OOF and 000. 8 culture plates were placed into MGC-450BP light incubator (Shanghai Yiheng Scientific Instruments Co., Ltd) for germination. The cultivated conditions were set as $12 \mathrm{~h}$ light $\left(25^{\circ} \mathrm{C}\right)$ and $12 \mathrm{~h}$ non-light $\left(15^{\circ} \mathrm{C}\right)$. Daily watering $(50 \mathrm{ml})$ was necessary during the cultivation.

Table 1

Experimental design of groups under salinity $(S)$, artemisinin $(A)$ and freezethaw (F) stress

\begin{tabular}{|lllllllll|}
\hline & SAF & SOF & OAF & OOF & SAO & SOO & OAO & OOO \\
\hline Salinity & + & + & - & - & + & + & - & - \\
\hline Artemisinin & + & - & + & - & + & - & + & - \\
\hline Freeze-thaw & + & + & + & + & - & - & - & - \\
\hline
\end{tabular}

\subsection{Freeze-thaw stress treatment and sampling}

After having grown for 8d, the seedlings of Groups SAF, SOF, OAF and OOF were put into BPHJ-120A highlow-temperature test chamber (Shanghai Yiheng Scientific Instruments Co., Ltd) to perform a freeze-thaw cycle for a period of $14 \mathrm{~h}$, while other cultivated dishes of Groups SAO, SOO, OAO and OOO were 
maintained in light incubator under previous culture conditions. Initially, the cultivated dishes were placed in the chamber at $15{ }^{\circ} \mathrm{C}$ that closed to room temperature at night. Controlled precisely by program, the temperature decreased to $-5^{\circ} \mathrm{C}$ steadily at a speed around $0.04{ }^{\circ} \mathrm{C} / \mathrm{min}$, and then the temperature increased from -5 to $10{ }^{\circ} \mathrm{C}$ at a speed around $0.04{ }^{\circ} \mathrm{C} / \mathrm{min}$. After the freeze-thaw cycle being started, five parallel samples were taken at $2 \mathrm{~h}, 8 \mathrm{~h}$ and $14 \mathrm{~h}$ from 8 cultivated dishes at random according to the required amount of the measurement, the corresponding sampling temperature was $10,-5,10^{\circ} \mathrm{C}$ respectively, recorded as $\mathrm{T} 1, \mathrm{~T} 2$, and T3.All the samples were firstly wrapped up with tin foil paper, secondly fixed in liquid nitrogen immediately for $50 \mathrm{~s}$ and finally put into the ultra-low-temperature freezer at $-80^{\circ} \mathrm{C}$ for storage in order to measure the content of MDA and soluble protein, SOD and POD activity. At the same time, fresh leaves were taken to determinate RC and WUE.

\subsection{Analysis}

\subsubsection{Relative electrical conductivity (RC)}

The relative electrical conductivity ( $\mathrm{RC}$ ) of leaves was measured before and after heat treatment to assess cell membrane damage, as described by $\mathrm{He}$ et al. with modifications (He et al, 2018). In brief, sampled leaves $(0.1 \mathrm{~g})$ were washed using deionized water for 3 times, cut into $0.5-1 \mathrm{~cm}$ pieces, and immersed in $10 \mathrm{ml}$ deionized water for $12 \mathrm{~h}$ at room temperature. Then, $\mathrm{RC}$ of the solution was measured using a conductivity meter (DDS-307, INESA Scientific Instrument Co., Ltd., Shanghai, China) and recorded as S1. After boiling the leaf samples for $30 \mathrm{~min}, \mathrm{RC}$ of the solution at room temperature was measured again and recorded as S2. A RC was calculated according toformula (1).

$$
\text { RWC }=\left(T_{w}-D_{W}\right) /\left(F_{W}-D_{W}\right) \times 100 \%
$$

\subsubsection{Soluble protein (SP)}

The soluble protein content was determined by the Coomassie brilliant blue method (Bradford 1976). For each replicate, $0.1 \mathrm{~g}$ leaves were homogenized with $5 \mathrm{ml}$ distilled water, and the mixture was centrifuged with a TDL-40B centrifuge (Anting Scientific Instrument Factory, Shanghai) at a speed of $3000 \mathrm{r} / \mathrm{min}$ for $10 \mathrm{~min}$. A $1 \mathrm{ml}$ sample of the supernatant was diluted in 5 times with $4 \mathrm{ml}$ distilled water, of which $1 \mathrm{ml}$ diluted supernatant was taken into a test tube with $5 \mathrm{ml}$ of Coomassie brilliant blue solution being added. After the mixed solution being shaken and placed for $2 \mathrm{~min}$, the absorbance at $595 \mathrm{~nm}$ was measured with a UV-6100 UV-visible spectrophotometer (Metash Co. Ltd) and translated into protein content using a calibration curve constructed with bovine serum albumin as standard.

\subsubsection{Malondialdehyde (MDA)}

Malondialdehyde (MDA) content was determined by the thiobarbituric acid method.(Gong et al, 2020)For each replicate, around $0.5 \mathrm{~g}$ leaves were homogenized with $5 \mathrm{ml} \mathrm{10 \%} \mathrm{trichloroacetic} \mathrm{acid} \mathrm{(TCA)} \mathrm{solution,}$ and the mixture was centrifuged at a speed of $4000 \mathrm{r} / \mathrm{min}$ for $10 \mathrm{~min}$. Then $2 \mathrm{ml}$ of the supernatant was taken and fixed with $2 \mathrm{ml} 0.6 \%$ thiobarbituric acid (TBA) solution. Mixtures was bathed in $99{ }^{\circ} \mathrm{C}$ water for 
$15 \mathrm{~min}$, then cooled quickly in $5 \mathrm{~min}$ and centrifuged again at a speed of $4000 \mathrm{r} / \mathrm{min}$ for $10 \mathrm{~min}$ with a TDL-40B centrifuge (Anting Scientific Instrument Factory, Shanghai). The absorbance of supernatant was measured at $532 \mathrm{~nm}, 600 \mathrm{~nm}$, and $450 \mathrm{~nm}$ with a UV-6100 UV-visible spectrophotometer (Metash Co. Ltd). The MDA concentration and MDA content were calculated according to formulas (2) and (3).

MDA concentration $(\mu \mathrm{mol} / \mathrm{L})=6.45 \times(\mathrm{D} 532-\mathrm{D} 600)-0.56 \times \mathrm{D} 450(2)$

$\mathrm{MDA}$ content $(\mu \mathrm{mol} / \mathrm{g})=\mathrm{cMDA} \times \mathrm{V}_{\mathrm{T}} / \mathrm{F}_{\mathrm{W}}$

where:

- D450, D532, D600 are the absorbance at 450nm, 532nm and 600nm, respectively.

- cMDA is MDA concentration ( $\mu \mathrm{mol} / \mathrm{L})$;

- VT is the volume of TCA solution (ml);

- FW is the fresh weight of seedlings (g).

\subsubsection{Superoxide dismutase (SOD) and peroxidase (POD) activities}

The activities of SOD and POD were determined with the SOD and POD kits provided by Nanjing Jiancheng Biological Institute. A parallel sample (around $0.25 \mathrm{~g}$ ) were randomly taken and homogenized with $5 \mathrm{ml}$ phosphate buffer on ice. After centrifugations at a speed of $3500 \mathrm{r} / \mathrm{min}$ and $3500 \mathrm{r} / \mathrm{min}$ with a TDL-40B centrifuge (Anting Scientific Instrument Factory, Shanghai), respectively for $10 \mathrm{~min}$, the supernatant was used for following measurements according to instructions of kits.

\subsubsection{Water use efficiency (WUE)}

The CIRAS-3 portable photosynthesis system(PP SYSTEMS, American) was used to determine the water use efficiency (WUE) in the barley seedling leaves. The $\mathrm{CO} 2$ buffer bottle is placed in a ventilated place when the photosynthesis measurement experiments were performed. The instrument is provided with a built-in LED light source. The setting parameter is PLC3 Universal Leaf Cuvette $(7 \times 25 \mathrm{~mm}$ window), 80\% for $\mathrm{H} 2 \mathrm{O}$ Reference, $300 \mu \mathrm{mol} \cdot \mathrm{m}-2 \cdot \mathrm{s}-1$ LED for Light Source, and 90, 0, 5, and 5 respectively for the RedGreen-Blue-White parameters in RGBW Control, besides, the temperature is controlled to $25 \pm 2{ }^{\circ} \mathrm{C}$.

\subsection{Statistical analysis}

The experiments were repeated five times, and the data were expressed as mean \pm standard error (SE) $(n=5)$, which statistically performed with R 3.3.1 statistical software (R Foundation for Statistical Computing, Vienna, Austria) for one-way analysis of variance (ANOVA) and Multivariate ANOVA. When the variables were uniform, the significance analysis of data was analyzed using Duncan model, otherwise using Games-Howell model (Warner 2013). All results were shown in bars in figures plotted by 
Origin 8.0 software. Different letters presented in figures indicated significant differences between different treatment groups at the same time.

\section{Result}

\subsection{Effect on the relative electrical conductivity (RC) of seedlings}

It can be seen from Fig. 1a that exposure of highland barley to stresses caused a stress-dependent increase in relative electrical conductivity $(\mathrm{RC})(P<0.05)$, and the maximum increase was observed under the compound stresses of salinity, artemisinin and freeze-thaw. Exposure of barley leaves to freeze-thaw stress exacerbated the effects and the RC in these plants at T3 represented 83.34\% (Group SAF), 102.51\% (Group SOF), $64.17 \%$ (Group OAF) and $11.88 \%$ (Group OOF) higher than that at $\mathrm{T} 1(P<0.05)$, while the treatment groups that not subjected to freeze-thaw stress represented no significant change in T1-T3. Furthermore, freeze-thaw stress significantly increased the RC in barley leaves of the four treatment groups (SA*, SO*, OA* and $\mathrm{OO}^{*}$ ) after treatment with freeze-thaw (Status $* * \mathrm{~F}$ ) as compared to Status $* * 0$ (Fig. 1b).The results above indicated that freeze-thaw, salinity and artemisinin stress had independent effects on RC, but had a significant synergistic relationship with each other.

\subsection{Effect on the soluble protein (SP) content of seedlings}

Figure 2a depicted that, under non-freeze-thaw conditions, artemisinin stress decreased soluble protein (SP) content as compared to control, however, there were no differences in SP content between control and treated plants under salinity stress. Simultaneous with the above changes, the SP content of leaves increased initially and then decreased in the groups treated with freeze-thaw stress, among which, SP content of Group SAF and SOF reduced $36.1 \%$ and $15.6 \%$ after treatments with freeze-thaw respectively, while Group OAF and OOF increased $47.7 \%$ and $102.9 \%$ respectively. It can be observed from Fig. $2 \mathrm{~b}$ that the SP content of leaves in Group SA* decreased, while that in Group SO*, OA* and OO* increased, indicating that freeze-thaw had a significant synergistic relationship with artemisinin stress and salinity stress respectively $(P<0.05)$, and that the group under salinity and artemisinin conditions could not synthesize more SP by itself to resist the adversity induced by freeze-thaw stress.

\subsection{Effect on malondialdehyde (MDA) content of seedlings}

The malondialdehyde (MDA) content of leaves in groups treated with stressincreased by $16.29 \%$ $82.62 \%$ compared to the control (Fig. 3a). Under non-freeze-thaw conditions, no significant change of MDA content in barley leaves was observed within 14-hour cultivation, while artemisinin stress increased the MDA content of leaves $(P<0.05)$. Meanwhile, the MDA content of the barley seedlings at lowest temperature (T2) in Groups SAF, SOF, OAF and OOF respectively represented $23.8 \%, 17.9 \%, 19.7 \%$ and $34.7 \%$ higher than that at the initial temperature $(\mathrm{T} 1)(P<0.05)$. Fig. $3 \mathrm{~b}$ suggested that the slope of Group $\mathrm{SO}$ * was the same as that of Group 00*,and the similar relationship was observed between Groups SA* 
and $\mathrm{OA}^{*}$. That is, the freeze-thaw stress and salinity stress are independent regardless of whether the artemisinin stress existed or not.

\subsection{Effect on superoxide dismutase (SOD) activity of seedlings}

As shown in Fig. 4a, at T1, freeze-thaw treatments reduced the SOD activity of the barley seedling leaves compared to the control(Group ${ }^{* *} 0$ ). During the freeze-thaw cycle, the SOD activity in the barley seedlings leaves increased by $10.0 \%$ (SAF), $27.0 \%$ (SOF), $11.3 \%$ (OAF) and $18.7 \%$ (OOF). Fig. $4 \mathrm{~b}$ depicted the increase in SOD activity of Group $\mathrm{SA}^{*}, \mathrm{SO}^{*}, \mathrm{OA}^{*}$ and $\mathrm{OO}$, indicating that freeze-thaw stress had an interaction relationship with artemisinin stress $(P<0.05)$. In addition, the increase in SOD activity of Group SO* after freeze-thaw treatment was more than that of Group 00*, while the increase in SOD activity of Group SA* after freeze-thaw treatment was less than that of Group $O A^{*}$, which demonstrated that under the artemisinin condition, freeze-thaw stress had an interaction relationship with salinity stress as well $(P<0.05)$.

\subsection{Effect on peroxidase (POD) activity of seedlings}

There was no significant change in POD activity of leaves in groups growing under non-freeze-thaw conditions within T1-T3 (Fig. 5a). However, the effects of freeze-thaw on POD activity of the barley seedling leaves were evident after 14-hour exposure, which was represented as a downward trend during freeze-thaw cycle. As can be observed in Fig. 5b, after 14-hour exposure of freeze-thaw stress, except for the increase in POD activity in the SA* treatment group, the POD activity in $\mathrm{SO}^{*}, \mathrm{OA}^{*}$ and $\mathrm{OO}^{*}$ treatments all decreased to varying degrees,proving that freeze-thaw stress had a very significant interaction relationship with artemisinin stress and salinity stress respectively $(P<0.01)$.

\subsection{Effect on water use efficiency (WUE) of seedlings}

The water use efficiency (WUE) of barley seedling leaves growing in the presence of freeze-thaw stress decreased significantly during the thawing process (T2-T3) and was much lower than that growing under non-freeze-thaw conditions (Fig. 6a). The maximum reduction of WUE was recorded in barley seedlings of Group SAF (-189.9\%), followed by Group SOF (-154.9\%) and Group OAF (-154.1\%), and the Group OOF decreased the least by $-127.6 \%$ with respect to the WUE at T1. We could see from Fig. $6 \mathrm{~b}$ that the decreasing slope of Group OA* was the same as Group 00*, which might indicate that the freeze-thaw stress and the artemisinin stress are independent in the conditions of non-salinity stress. On the contrary, the decreasing slope of Group SA* differed from that of Group SO*, indicating that the freeze-thaw stress had significant synergistic relationship when exposed tosalinity stress $(P<0.05)$. Similarly, the freezethaw stress had a synergistic relationship with salinity stress no matter whether artemisinin stress was applied or not $(P<0.05)$, which could be demonstrated in Fig. 6b.

\section{Discussion}


It is well-documented that the application of compounds with allelopathic effectscould result in the damage to the function of plant cell membranes (Romagni et al, 2000). Induced by adversity stress, excessive reactive oxygen species (ROS) in cells could lead to membrane peroxidation, resulting from which, the structure of plant cell membranes was destroyed, leading to an increasing RC (Wang et al, 2021). RC is characterized by the selective permeability function of cell membranes and, for this reason, $\mathrm{RC}$ is highly susceptible to environmental stresses. In this present study, single or combined stress of artemisinin, salinity and freeze-thaw increased the RC of barley seedlings compared to the control, which suggested that, to a certain extent, the cell membrane of leaves may have been destroyed under environmental stresses. Therefore, the reason for increased RC was the electrolyte extravasation due to the loss of selective permeability caused by damaged cell membrane. This may attribute to the accumulation of excessive ROS in the cells (Apel and Hirt, 2004). The maximum increase of RC was observed in Group SA* that followed by Group $\mathrm{OA}^{*}$, Group SO* and Group OO*, proving that the combined stresses of salinity and artemisinin reduced the regulating ability of plants and that salinity stress was more harmful to cells than artemisinin stress. Additionally, MDA increased when the cell membrane was damaged by ROS, and its content could reflect the severity of the stress which the plant subjected to (Karagoz et al, 2018). Previous researches proved that MDA content would increase in those plants exposed to freeze-thaw stress (Balestrasse et al, 2010), and similar results were observed in our study. During the freeze-thaw stress cycle, compared with the single freeze-thaw group, both salinity and artemisinin stress significantly increased the MDA content in the barley leaves, indicating that the two stresses further exacerbatethe damage (Babula et al, 2009).

Allelochemicals appear to alter a variety of physiological processes and it is difficult to separate the primary from the secondary effects. Several action modes have been suggested, including direct inhibition of protein synthesis (Yan et al, 2018) and degradation of macromolecular proteins (Liu et al, 2008). From the results, it is clear that artemisinin stress reduced the SP content of barley leaves, attributing to allelochemicals initially reduce the incorporation of certain amino acids that are raw materials of protein synthesis, consequently, the rate of protein synthesis reduced (Baziramakenga et al, 1997). Under freeze-thaw condition, the SP content of the seedlings increases significantly as the temperature dropping down, which is consistent with previous studies, in which researchers demonstrated that the accumulation of SP induced by the decreasing temperature might reduce the osmotic potential of cells (Airaki et al, 2012), improving the water retention capacity, and eventually, the damage of freeze-thaw stress to plants was relieved (Bao et al, 2019b). The SP content of seedling leaves in the present of salinity stress (SO*) increased more, while that of leaves in the present of artemisinin stress $\left(\mathrm{OA}^{*}\right)$ increased less compared to control $\left(\mathrm{OO}^{*}\right)$, indicating that the salinity stress may improve the resistance of highland barley to freeze-thaw stress to a certain extent, while artemisinin stress might make highland barley more sensitive to freeze-thaw stress, and the combined stress of salinity and artemisinin may weaken the osmotic adjustment ability of highland barley.

Changes in the antioxidant enzymes activity are good indicators of improving their resistance to adversity. In the present study, it was observed that SOD activity of the leaves in groups in the exposure of freeze-thaw stress was significantly enhanced (Meng et al, 2016). Plants can produce a variety of by- 
products of aerobic metabolism in the process of physiological metabolism. When entering the plant, oxygen initially produces $02-$, which would be converted into ROS including $\mathrm{H} 2 \mathrm{O} 2$ and $-\mathrm{OH}$. It has been proved that excessive ROS can induce cell apoptosis (Gechev et al, 2006). In the early stage of stress, the antioxidant enzymes activity of wheat seedlings increased to resist the adversity, however, as the stress exacerbates, the antioxidant enzymes activities would decrease then, aggravating the membrane lipid peroxidation (Bao et al, 2019a). Similarly, compared with the control, the SOD activity of the highland barley seedlings in the present of salinity and artemisinin decreased, and POD activity increased. Continuous application of salinity and artemisinin reduced SOD enzyme activity, leading to the accumulation of ROS and the increasing severity of membrane lipid peroxidation, and consequently, increasing the MDA content. After freeze-thaw stress being added, the SOD activity of highland barley seedlings increased, and the POD activity decreased, which might be SOD enzyme activity plays an important role in improving the tolerance of barley to freeze-thaw (Bian et al, 2020).

Water is one of the most important materials for photosynthesis, and leaf WUE described the relationship between the formation of organic matter and the water usage during plants growth (Liang et al, 2013). Under non-freeze-thaw conditions, no changes were observed in the WUE of barley leaves, and there was no significant difference between those groups. The results above indicated that a normal WUE of barley still maintained although it is under the stress of salinity and artemisinin. On the contrary to the former results, the WUE of barley in freeze-thaw treatment groups initially decreased little and subsequently dropped to a negative value during the thawing stage. Perhaps because the barley slowly adapted to adversity as the temperature dropped and the low temperature inhibited the synthesis of chlorophyll, which effected and reduced the photosynthetic rate. This was confirmed in Bao's research (Bao et al, 2020). Subsequently, in the process of thawing, the WUE represented a negative value, which might due to the fact that the newly synthesized organic substances in photosynthesis can no longer meet the energy demand of plant respiration under the low temperature environment.

\section{Conclusions}

In summary, as an important crop on the Qinghai-Tibet Plateau, barley has equipped with great resistance to the freeze-thaw environment within the long-term evolution. However, improper agricultural production measures have led to increasingly serious salinization. Besides, the artemisinin, an allelochemical, is widely distributed on the Qinghai-Tibet Plateau. These abiotic stresses mentioned above would lead to complex physiological changes in plants. In the present study, RC and MDA content in barley leaves were increased due to the application of salinity, artemisinin and freeze-thaw stress, among which, compound stresses caused the most serious damage to plant cell membrane permeability. Moreover, the SP content of barley present an initial increasing and a subsequent decline during freeze-thaw stress, suggesting that the exposure of barley to artemisinin stress inhibited the self-osmotic regulation when subjected to freeze-thaw stress at the same time. When it comes to antioxidant enzymes activity and WUE, freezethaw had significant synergistic relationship with artemisinin stress and salinity stress respectively. On the whole, freeze-thaw stress presented a synergistic stress relationship with salinity and artemisinin stress. Though the resistance characteristics of plants under one freeze-thaw cycle were studied in this 
paper, in view of multiple freeze-thaw cycles in nature, one important future direction of physiological responses to freeze-thaw stress is studying the different resistance characteristics of plant between under one freeze-thaw cycle and under multiple freeze-thaw cycles.

\section{Declarations}

\section{ACKNOWLEDGMENTS}

This work was sponsored by the National Natural Science Foundation of China (Grant Nos. 31772669 and 32071874) $\varangle$ Key Projects of Science and Technology Development Plan of Jilin Province (Grant No.20210203001SF), Interdisciplinary Project of Jilin University『Grant No. JLUXKJC2020107囚and the 111 Project (B16020)

\section{FUNDING}

This work was sponsored by the National Natural Science Foundation of China (Grant Nos. 31772669 and 32071874) बKey Projects of Science and Technology Development Plan of Jilin Province (Grant No.20210203001SF), Interdisciplinary Project of Jilin University『Grant No. JLUXKJC2020107囚and the 111 Project (B16020)

\section{COMPETING INTERESTS}

The authors have no relevant financial or non-financial interests to disclose.

\section{AUTHOR CONTRIBUTIONS}

When writing statement, Zhang Wei and Tang Wenyi designed research. Zhang Wei, Tang Wenyi, Xiao Jing, Dai Gejun, Liu Jiapeng and Wang Zhao conducted experiments. Zhang Wei and Dai Gejun wrote the main manuscript and Xiao Jing, Liu Jiapeng and Wang Zhao prepared figures 1-6.All authors reviewed the manuscript.

\section{AVAILABILITY OF DATA AND MATERIALS}

All relevant data generated or analysed during this study are included in this published article. Other data are not applicable.

\section{References}

1. Airaki M, Leterrier M, Mateos R M, et al. Metabolism of reactive oxygen species and reactive nitrogen species in pepper (Capsicum annuum L.) plants under low temperature stress [J]. Plant Cell and Environment,2012,35(2): 281-295.

2. Apel, K., Hirt H. Reactive oxygen species: metabolism, oxidative stress, and signal transduction. Annu. Rev. Plant Biol. 2004, 55: 373-399. 
3. Arfan $\mathrm{M}$ et al. (2019) CO2-EXCHANGE AND CHLOROPHYLL FLUORESCENCE RESPONSES OF FORAGE GRASSES DURING SALT STRESS AND RECOVERY IN QINGHAI-TIBETAN PLATEAU (QTP) Pakistan Journal Of Botany 51:1615-1628.

4. Babula, P., Adam, V., Kizek, R., Sladky, Z., Havel, L.: Naphthoquinones as allelochemical triggers of programmed cell death. - Environ. Exp. Bot. 2009, 65: 330-337.

5. Balestrasse K B, Tomaro M L, Batlle A, et al. The role of 5-aminolevulinic acid in the response to cold stress in soybean plants [J]. Phytochemistry,2010,71(17-18):2038-2045.

6. Bao $\mathrm{G}$ et al. (2019b) PHYSIOLOGICAL RESPONSES OF ALFALFA SEEDLINGS TO FREEZE-THAW CYCLES AND ALKALINE SALT STRESS Fresenius Environmental Bulletin 28:4114-4122.

7. Bao G, He F, Chen W, Sun J, Ding X. Physiological effects of different concentrations of chloride deicing salt and freeze-thaw stress on Secale cereale L. seedlings[J]. Journal of Plant Growth Regulation, 2019a

8. Bao G. Z., Tang W. Y., Zhou S. J., Liu B. J., Wu Z. Z., Jin D. X., Bian W. J., Zhu S. N.Photosynthesis Characteristics of Tall Fescue under Snow-Melting Agent, Acid Precipitation and Freeze-Thaw Stress[J]. Russian Journal of Plant Physiology, 2020.

9. 40 Baziramakenga R, Leroux GD, Simard RR, Nadeau P. 1997. Allelopathic effects of phenolic acids on nucleic acid and protein levels in soybean seedlings. Canadian Journal of Botany 75, 445-450.

10. Bertrand A, Bipfubusa $M$, Castonguay $Y$, et al. A proteome analysis of freezing tolerance in red clover (Trifolium pratense L.)[J]. BMC Plant Biology, 2016, 16, 65.

11. Bian W, Bao GZ, Liao JF et al. Comparative study on physiological response characteristics of white clover to chloride salt and calcium-magnesium acetate (CMA) deicing agents under freeze-thaw stress[J] Grassland Science, 2020, 66, 95-101.

12. Bradford MM. 1976. A rapid, sensitive method for the quantitation of microgram quantities of protein utilizing the principle of protein-dye binding. Annals of Biochemistry 72, 248-254.

13. Che Z, Wang J, Li JS. Effects of water quality, irrigation amount and nitrogen applied on soil salinity and cotton production under mulched drip irrigation in arid Northwest China[J]. Agricultural Water Management, 2021, 247, 106738.

14. Domisch T, Martz F, Repo T, et al. Let it snow! Winter conditions affect growth of birch seedlings during the following growing season[J]. Tree Physiology, 2019, 39(4), 544-555.

15. Gechev, T.S., Breusegem, F.V., Stone, J.M., Denev, I., Laloi, C.: Reactive oxygen species as signals that modulate plant stress responses and programmed cell death. - Bioessays 28: 1091-1101, 2006.

16. Gong Z, Chen WW, Bao GZ, et al. Physiological response of Secale cereale L. seedlingsunder freezing-thawing and alkaline salt stress [J]. Environmental Science and Pollution Research, 2020, 27, 1499-1507.

17. Guo T. Long-Term Changes and Variability of Ecologically-Based Climate Indices along an Altitudinal Gradient on the Qinghai-Tibetan Plateau[J]. Climate, 2021, 9(1), 1. 
18. He AL, Niu SQ, Zhao Q, Li YS, Gou JY, Gao HJ, et al. Induced salt tolerance of Perennial ryegrass by a novel bacterium strain from the rhizosphere of a desert shrub Haloxylonammodendron. Int J Mol Sci. 2018; 19: 469-472.

19. Herrmann S, Jessing KK, Jorgensen NOG, et al. Distribution and ecological impact of artemisinin derived from Artemisia annua L. in an agricultural ecosystem[J]. Soil Biology \& Biochemistry, 2013, $57,164-172$.

20. Hussain MI, Reigosa MJ. Evaluation of Herbicide Potential of Sesquiterpene Lactone and Flavonoid: Impact on Germination, Seedling Growth Indices and Root Length in Arabidopsis Thaliana[J]. 2014, 46(3), 995-1000.

21. Karagoz H, Cakmakci R, Hosseinpour A, Kodaz S. ALLEVIATION OF WATER STRESS AND PROMOTION OF THE GROWTH OF SUGAR BEET (BETA VULGARIS L.) PLANTS BY MULTI-TRAITS RHIZOBACTERIA Applied Ecology and Environmental Research, 2018, 16:6801-6813.

22. of sticky maize with fertigation[J].Agricultural Water Management,2013,116:242-247.

23. Liu J, Wu YH, Yang JJ, Liu YD, Shen FF (2008) Protein degradation and nitrogen remobilization during leaf senescence. J Plant Biol 51: 11-9.

24. Meng X, Zhao Q, Jin Y, et al. Chilling-responsive mechanisms in halophyte Puccinelliatenuiflora seedlings revealed from proteomics analysis [J]. Journal Of Proteomics, 2016, 143, 365-381.

25. Min K, Chen K, Arora R. A metabolomics study of ascorbic acid-induced in situ freezing tolerance in spinach (Spinacia oleracea L.)[J]. Plant Direct, 2020, 4(2), e00202.

26. Qian YPL, An YQ, Chen S, et al. Characterization of Qingke Liquor Aroma from Tibet[J]. Journal of Agricultural And Food Chemistry, 2019, 67(50), 13870-13881.

27. RomagniJG,AllenSN,Dayan FE. Allelopathic effects of volatile cineoles on two weedy plant[J]. J Chem Ecol,2000,26(1):303-314.

28. Wang XQ, Chen RS, Liu GH, et al. Spatial distributions and temporal variations of the near-surface soil freeze state across China under climate change[J]. Global and Planetary Change, 2019, 172, 15058.

29. Wang Z, Zhang Q, Qin J, et al. OsLEA1a overexpression enhances tolerance to diverse abiotic stresses by inhibiting cell membrane damage and enhancing ROS scavenging capacity in transgenic rice[J]. Functional Plant Biology, 2021, FP20231.

30. $₫ \mathrm{X} \bigotimes$ Warner, R. M. (2013). Applied statistics: From bivariate through multivariate techniques(2nd ed.). Thousand Oaks, Calif: SAGE Publications.

31. Yan ZQ, Wang DD, Cui HY, et al. Effects of artemisinin on root gravitropic response and root system development in Arabidopsis thaliana[J]. Plant Growth Regulation, 2018, 85(2), 211-220.

32. Yan ZQ, Wang DD, Ding L, et al. Mechanism of artemisinin phytotoxicity action: Induction of reactive oxygen species and cell death in lettuce seedlings[J]. Plant Physiology and Biochemistry, 2015, 88, 53-59. 
33. Ye L, Qian J, Jin Song, et al. Algicidal effects of four Chinese herb extracts on bloom-forming Microcystis aeruginosa and Chlorella pyrenoidosa[J]. Environmental Technology, 2014, 35(9), 11501156.

34. Zhang P, Duo TQ, Wang FD, et al. De novo transcriptome in roots of switchgrass (Panicum virgatum L.) reveals gene expression dynamic and act network under alkaline salt stress[J], BMC Genomics, 2021, 22(1), 82.

\section{Figures}

Figure 1

(a) Changes in relative electrical conductivity of barley seedling leaves under different treatment conditions. (b) The interaction relationship between freeze-thaw stress, salinity stress and artemisinin stress. (T1-T3 represents the $2^{\text {nd }}, 8^{\text {th }}$, and $14^{\text {th }}$ h sampling points in freeze-thaw cycle, and the corresponding temperatures are $10,-5$, and $10^{\circ} \mathrm{C}$ respectively. S: salinity stress, $\mathrm{A}$ : artemisinin stress, $\mathrm{F}$ : freeze-thaw stress, O: not subject to the certain stress, *: including whether subject to the stress or not. Different letters indicate the difference between different treatment groups at the same time $(P<0.05)$.)

\section{Figure 2}

(a) Changes in the soluble protein content of barley seedling leaves under different treatment conditions.

(b) The interaction relationship between freeze-thaw stress, salinity stress and artemisinin stress. (T1-T3 represents the $2^{\text {nd }}, 8^{\text {th }}$, and $14^{\text {th }} \mathrm{h}$ sampling points in freeze-thaw cycle, and the corresponding temperatures are $10,-5$, and $10^{\circ} \mathrm{C}$ respectively. S: salinity stress, A: artemisinin stress, F: freeze-thaw stress, O: not subject to the certain stress, *: including whether subject to the stress or not. Different letters indicate the difference between different treatment groups at the same time $(P<0.05)$.)

\section{Figure 3}

(a) Changes in malondialdehyde (MDA) content of barley seedling leaves under different treatment conditions. (b) The interaction relationship between freeze-thaw stress, salinity stress and artemisinin stress. (T1-T3 represents the $2^{\text {nd }}, 8^{\text {th }}$, and $14^{\text {th }} \mathrm{h}$ sampling points in freeze-thaw cycle, and the corresponding temperatures are $10,-5$, and $10^{\circ} \mathrm{C}$ respectively. S: salinity stress, A: artemisinin stress, $\mathrm{F}$ : freeze-thaw stress, O: not subject to the certain stress, *: including whether subject to the stress or not. Different letters indicate the difference between different treatment groups at the same time $(P<0.05)$.) 


\section{Figure 4}

(a) Changes in superoxide dismutase (SOD) activity of barley seedling leaves under different treatment conditions. (b) The interaction relationship between freeze-thaw stress, salinity stress and artemisinin stress. (T1-T3 represents the $2^{\text {nd }}, 8^{\text {th }}$, and $14^{\text {th }} \mathrm{h}$ sampling points in freeze-thaw cycle, and the corresponding temperatures are $10,-5$, and $10^{\circ} \mathrm{C}$ respectively. S: salinity stress, $\mathrm{A}$ : artemisinin stress, $\mathrm{F}$ : freeze-thaw stress, O: not subject to the certain stress, *: including whether subject to the stress or not. Different letters indicate the difference between different treatment groups at the same time $(P<0.05)$.)

\section{Figure 5}

(a) Changes in peroxidase (POD) activity of barley seedling leaves under different treatment conditions. (b) The interaction relationship between freeze-thaw stress, salinity stress and artemisinin stress. (T1-T3 represents the $2^{\text {nd }}, 8^{\text {th }}$, and $14^{\text {th }} \mathrm{h}$ sampling points in freeze-thaw cycle, and the corresponding temperatures are $10,-5$, and $10^{\circ} \mathrm{C}$ respectively. S: salinity stress, A: artemisinin stress, F: freeze-thaw stress, 0: not subject to the certain stress, *: including whether subject to the stress or not. Different letters indicate the difference between different treatment groups at the same time $(P<0.05)$.)

Figure 6

(a) Changes in water use efficiency (WUE) of barley seedling leaves under different treatment conditions. (b) The interaction relationship between freeze-thaw stress, salinity stress and artemisinin stress. (T1-T3 represents the $2^{\text {nd }}, 8^{\text {th }}$, and $14^{\text {th }} \mathrm{h}$ sampling points in freeze-thaw cycle, and the corresponding temperatures are $10,-5$, and $10^{\circ} \mathrm{C}$ respectively. S: salinity stress, A: artemisinin stress, F: freeze-thaw stress, O: not subject to the certain stress, *: including whether subject to the stress or not. Different letters indicate the difference between different treatment groups at the same time $(P<0.05)$.) 